American Journal of Agricultural and Biological Sciences 3 (1): 299-314, 2008

ISSN 1557-4989

(C) 2008 Science Publications

\title{
Polymer in Agriculture: a Review
}

\author{
Francesco Puoci, Francesca Iemma, Umile Gianfranco Spizzirri, Giuseppe Cirillo, \\ Manuela Curcio and Nevio Picci \\ Dipartimento di Scienze Farmaceutiche, Università della Calabria \\ Arcavacata di Rende (CS) 87036, Italy
}

\begin{abstract}
In agricultural field, polymers are widely used for many applications. Although they were used, in the first time, just as structural materials (inhert polymers), in the last decades functionalized polymers revolutionized the agricultural and food industry with new tools for several applications. Smart polymeric materials and smart delivery systems helped the agricultural industry to combat viruses and other crop pathogens, functionalized polymers were used to increase the efficiency of pesticides and herbicides, allowing lower doses to be used and to indirectly protect the environment through filters or catalysts to reduce pollution and clean-up existing pollutants. This report will review the key aspects of used polymers in agricultural area, highlighting current research in this field and the future impacts they may have.
\end{abstract}

Keywords: Fuctional polymers, smart materials.

\section{INTRODUCTION}

Polymers are an important part in everyday life; products made from polymers range from sophisticated articles, such as biomaterials (prosthetic hip and knee joints), to aerospace materials. One of the reasons for the great popularity exhibited by polymers is their ease of processing. Polymer properties can be tailored to meet specific needs by varying the "atomic composition" of the repeat structure and by varying molecular weight. The flexibility can also be varied through the presence of side chain branching and according to the lengths and the polarities on the side chains. The degree of crystallinity can be controlled through the amount of orientation imparted to the polymers during processing, through copolymerization, by blending with other polymers, and via the incorporation (via covalent and non-covalent interactions) of an enormous range of compounds.

In agricultural field, polymers are also widely used for many applications ${ }^{[1]}$. Although they were used, in the first time, just as structural materials for creating a climate beneficial to plant growth (inhert polymers), in the last decades functionalized polymers revolutionized the agricultural and food industry with new tools for the molecular treatment of diseases, rapid disease detection, enhancing the ability of plants to absorb nutrients etc..
Smart polymeric materials and smart delivery systems helped the agricultural industry combat viruses and other crop pathogens, functionalized polymers were used to increase the efficiency of pesticides and herbicides, allowing lower doses to be used and to protect the environment indirectly through filters or catalysts to reduce pollution and clean-up existing pollutants $^{[2-4]}$. This report will review the key aspects of used polymers in agricultural area, highlighting current research in this field and the future impacts they may have.

\section{BIODEGRADABLE POLYMERS IN THE AGRICULTURAL FIELD}

In the last decade, one of the problems affecting the environment has been the increased use of plastic materials and their subsequent disposal. Plastics have been used in innumerable applications with little consideration for their ultimate disposability. Conventional polymers, such as polyethylene and polypropylene persist for many years after disposal. Built for the long haul, these polymers seem inappropriate for applications in which plastics are used for short time periods and then disposed. Furthermore, plastics are often soiled by food and other biological substances. In other words, the resistance of synthetic

Corresponding Author: Francesco Puoci ,Dipartimento di Scienze Farmaceutiche, Università della Calabria Edificio Polifunzionale Arcavacata di Rende (CS) 87036, Italy, Tel. +390984493151 Fax. +390984493298 
polymers to the action of living systems is becoming more and more problematic in several domains where are used for a limited period of time before becoming waste. Among the various possible routes to eliminate polymeric wastes, biodegradation and biorecycling via bioassimilation are regarded as attractive solutions for environmental protection, when incineration is not feasible because it is a source of unacceptable pollution.

Biodegradable polymers (BPs) have increasingly been used such as plastics substitutes for several applications in the agriculture field ${ }^{[5-7]}$. BPs, disposed in bioactive environments, degrade by the enzymatic action of microorganisms such as bacteria, fungi, and algae and their polymer chains may also be broken down by non enzymatic processes such as chemical hydrolysis. Unfortunately, in the majority of cases, the properties of natural polymers do not fit the needs of specific applications, and blending with synthetic polymers is a route largely used to gain the desired properties $^{[8,9]}$. Convenient candidates for those applications are natural polymers such as agar, starches, alginates, pectins and cellulose derivatives, along with synthetic biodegradable polymers, such as polycaprolactone, polylactide and poly (vinyl alcohol). ${ }^{[10-13]}$ (Fig. 1).
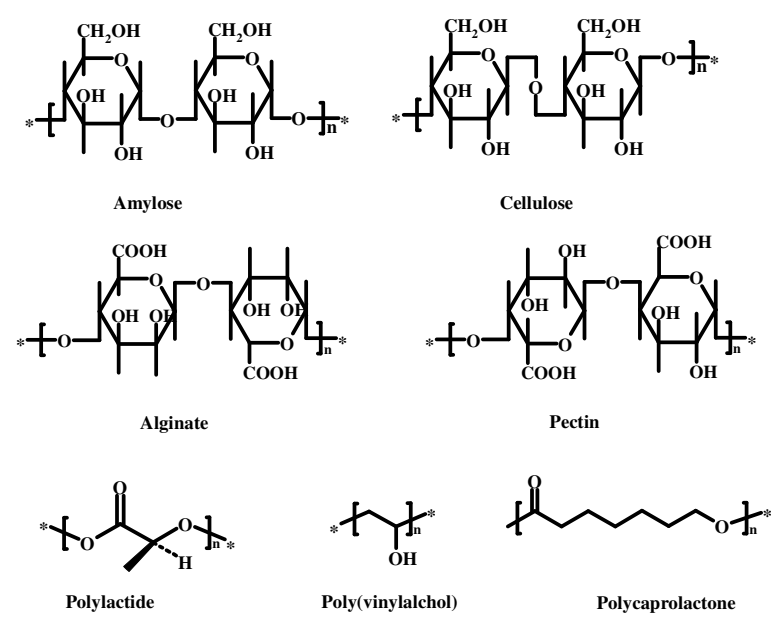

Fig. 1: Natural and synthetic biodegradable polymers

Starch is an inexpensive, annually renewable material derived from corn and other crops. All starches contain amylose and amylopectin, at ratios that vary with the starch source. This variation provides a natural mechanism for regulating starch material properties. Starch-based biodegradable polymers can be produced by blending or mixing them with synthetic polymers.
By varying the synthetic blend component and its miscibility with starch, the morphology and hence the properties can be regulated easily and efficiently. ${ }^{[14]}$ Blends containing thermoplastic starch (non crystalline starch) may be blended or grafted with biodegradable polyesters, such as polycaprolactone, to increase flexibility and resistance to moisture. Blends, mainly formed into films and sheets, with more than $85 \%$ starch are used for foaming and injection molding ${ }^{[15]}$. By mixing thermoplastic starch with cellulose derivatives, rigid and dimensionally stable injectionmolded articles result. Chemically modified plant cellulose is used in a remarkably diverse set of applications. For example, cellulose acetate is used in many common applications, including toothbrush handles and adhesive tape backing. Studies in simulated compost environments revealed that cellulose acetates with degrees of substitution of up to 2.5 are biodegradable. A decrease in the degree of substitution of cellulose acetate from 2.5 to 1.7 results in a large increase in the rate of their biodegradation ${ }^{[16]}$. An important application of these biodegradable polymers is their employment in solarization process. Indeed, one of the problems afflicting agricultural production is the presence of parasites in the soil that, along with spontaneous weeds, take away nourishment from the soil. In the past, the elimination of parasites and seeds of undesirable plants, before a new sowing, was performed through fumigation with methyl bromide, which has been indefinitely banned for its toxicity. In the 1970s, a new approach, solarization, ${ }^{[17,18]}$ which involves covering the soil to be reconditioned with polymeric films, was introduced. The polymeric films for this application have to be mechanically resistant, transparent to visible light, and opaque to infrared radiation. The optical properties are important because, during the day, visible radiation, which passes through the film, warms the soil. During the night, when the soil cools by emitting infrared radiation, the film, which is impermeable to infrared radiation, traps it and thus prevents heat loss. Actually, a film with these optical properties has a micro greenhouse effect on the soil. This technique is largely used today, particularly at those latitudes with temperate climate. It makes use of low-density polyethylene with fillers, such as phosphates, that increase the opacity to infrared radiation. Solarization guarantees the decontamination of soils assigned to insemination within 4-6 weeks. At the end of the treatment, the problem of the removal and disposal of films has to be resolved. Films based on synthetic polymers have to be treated as special waste with additional costs. Moreover, there are several 
problems related to environmental pollution for all films that, in violation of the law, are burned after their use. A biodegradable films, made of natural polymers, for solarization offers the advantage that it does not have to be removed from the soil after they are used. In literature film for solarization containing alginates, poly(vinyl alcohol) and glycerol are reported. ${ }^{[19,20]}$ Alginates are water-soluble linear copolymer, containing $\alpha$-gluronic acid and $\beta$-mannuronic acid units, present in seaweed. ${ }^{[21,22]}$ The proposed materials were characterized in terms of the mechanical parameters and optical properties. The films were transparent in the visible region and opaque in the infrared region to ensure a micro greenhouse effect on the soil.

\section{POLYMERS FOR AGROCHEMICALS DELIVERY}

The use of conventional agrochemicals produce undesirable side-effects such as their incorporation into the food chain and the contamination of the surrounding environment. Persistent and less persistent herbicides are either inadequate because the first may be hazardous for humans care while the seconds necessitate of multiple applications with high exposure to operators and high cost. Polymer supported herbicides overcome the serious environmental problems of the conventional herbicides because they release the herbicide to the plant at a controlled rate in the quantities required over a specified period of time. There are two different approaches in combining the agrochemicals agents with polymeric materials: either by physical combination (encapsulation, or heterogeneous dispersion) to act as a rate controlling device, or by chemical combination to act as carrier for the agent. The choice of the best system to release the active agent is highly dependent on the biological and chemical properties of the agent and on its physicochemical interactions in the system. A wide and diverse range of polymer compositions have been used to fabricate agrochemicals delivery devices. Particular interest was focussed on polymers containing herbicides as pendant groups (Pentachlorophenol (PCP), 2,4-dichlorophenoxyacetic acid (2,4-D) and 4chloro-2-methylphenoxyacetic acid (CMPA). Materials able to release an herbicide in the soil, and at the same time, to increase the water retention by sandy soil, were realized linking covalently the herbicides to hydrophilic polymers. Hydrogels prepared from oligooxyethylene methacrylates were used to link CMPA via ionic and covalent interactions. Solaro and coworkers synthesized hydrophilic polymers based on different acrylates monomers such as diethylene glycol methacrylate (DEGMA), octaethylene glycol methacrylate (OEGMA), acrylamide (AA), tetraethylene glycol methacrylate (TEGMA) and N,N'methylenebisacrylamide (MNBA) as cross-linking agent in 2,4-D and CMPA release ${ }^{[23]}$. The same authors proposed a device characterized by Poly(methacrylic acid) cross-linked with divinylbenzene (DVB), derivatized with hydrazine, ethylene diamine and examethylendiamine followed by modification with the acid chlorides of 2,4-D and CMPA. Polymeric materials, which have properties of fertilizers and herbicides at the same time, were also obtained by binding 2,4-D derivatives (tartrate and glutarate) to hydrophilic polymers such as polyamides, polyureas, poly(Schiff base)s and polyesters and their release was investigated. Akelah et al. proposed one easy synthesis of polyamides containing pendant hydroxyl groups from diethyil-L-tartrate and various diamines ${ }^{[2]}$. The polymers obtained in this way represent a potentially bioactive degradable supports able to release an herbicid, 2,4-D, in the soil (Figure 2).

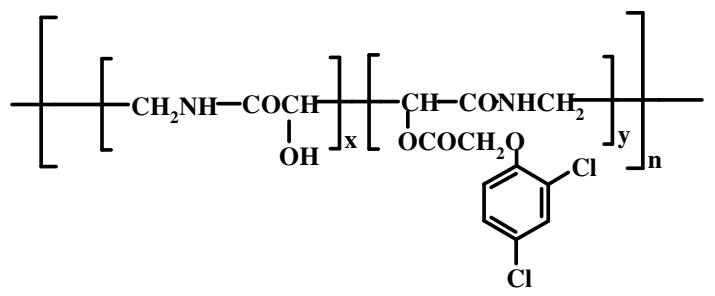

Fig. 2: 2,4-D derivatized polyamides.

The rate of release of 2,4-D from copolymers is dependent on the polymeric hydrophilicity, the $\mathrm{pH}$ and the temperature of medium. When the hydrophilicity increase the increased diffusion of medium in the polymer allow the attack upon the bonding group. The change in the $\mathrm{pH}$ and temperatures of the medium surrounding the polymers, showed an increase in the rate of the hydrolysis reaction. Cross-linked gel based on polyacrylamide derivatized with various diammine were also tested by Kenawy et al. in order to realize a slow release of chemically bounded $2,4-\mathrm{D}^{[24]}$ (Figure 3 ).
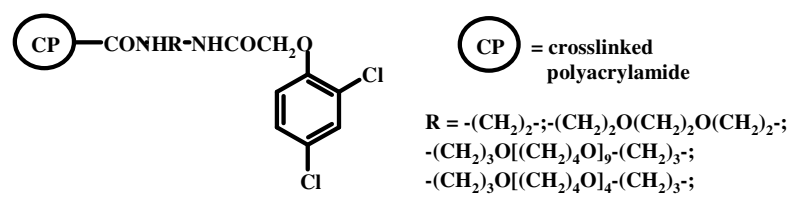

Fig. 3: 2,4-D chemically bounded to crosslinked polyacrylamide via various diamines. 
In vitro release experiments of 2,4-D herbicide from acrylamide-based gels suggested that the release rate is dependent on the $\mathrm{pH}$ of the medium; it was slower in acidic medium than in neutral or alkaline medium, and on the length of the side-chain spacer group (R). Instead 2,4,5-trichlorophenoxyacetic acid $(2,4,5-\mathrm{T})$ was encapsulated in a matrices based on starch-g-poly(vinyl alcohol) (starch-g-PVA). These ones were prepared by saponification of the corresponding poly(vinyl acetate) (starch-g-PVAc) with $\mathrm{NaOH}^{[25]}$. Starch-g-poly(butyl acrylate) was employed by Zhu et al. to encapsulate alkali-sensitive chemicals, such as carboxylic-containing herbicides. 2,4-D and 2,4,5-T were indeed efficiently encapsulated in the modified starch matrix and slow release behaviour was observed $^{[26]}$.

The Variation of grafting ratio, molar ratio of $\mathrm{NaOH}$ to acetate groups, herbicide content and particle size all influence the swellability and release rate. In particular increasing the grafting ratio, herbicide content, particle size or decreasing the molar ratio of $\mathrm{NaOH}$ to acetate, a slow release of herbicide was observed. Moreover the interaction between the hydroxyl group present on the matrices and the carboxylic-containing herbicides is favourable to slow release. Taki Et al.reported on a controlled delivery systems for the herbicide N-(3,4-dichlorophenyl)-N,Ndimethyl urea (Diuron) obtained by coprecipitation, in methylene chloride, using a supercritical antisolvent technique (SAS) which allows to load the herbicide in amorphous microparticles of a biodegradable polymer (L-polylactic acid, L-PLA) ${ }^{[27]}$. The SAS process involves the spraying of the solution composed of the solute and of the organic solvent into a continuous supercritical phase flowing cocurrently. The paper reports the first results of this work and concerns the influence of various process parameters upon the size and morphology of the particles formed, i.e. pressure, temperature, composition and flow rates. Successively other coating materials such as the poly(methyl methacrylate) (PMMA) were also tested but did not give convincing results for the researchers ${ }^{[28]}$. A biodegradable polymeric microspheres (Figure 4) based on ethyl cellulose loaded with 2,4-D were prepared by the emulsion solvent-evaporation technique to develop controlled release formulations that protect the herbicide from photodegradation and evaporation and to reduce the environment pollution ${ }^{[29]}$. In this work Elbahri et al. studied the process control parameters of microencapsulation and factors controlling the drug release like stirring speed, stabiliser concentration, polymer concentration, drug-polymer ratio, $\mathrm{pH}$ of external phase and internal phase solvent etc.. The drug entrapment can be improved especially by increasing polymer-solvent ratio or/and decreasing $\mathrm{pH}$ of continuous phase. The drug loading can increase from 7.7 to $27 \%$ by modifying the process parameters. Particle size can be controlled absolutely by stirring speed and polymer-solvent ratio.

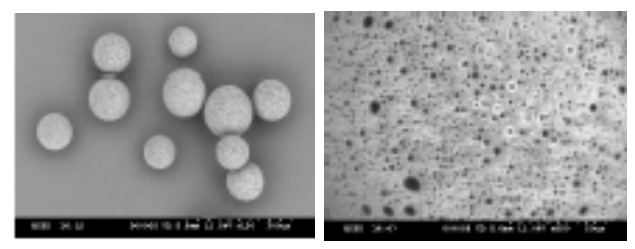

Fig. 4: SEM micrographs of ethylcellulose microspheres loaded by 2,4-D

The release rate depends strongly on the particle size because that drug liberation is governed by diffusion process throughout ethylcellulose microspheres. Microcapsules of natural rubber, PE, copolymers of $\mathrm{VC}$-acrylic acid esters and copolymers of cyclopentadiene with a glyceryl ester of an unsatured fatty acid were also prepared by film-forming on the fertilizer granules in order to obtain a water insoluble coating around the fertilizer which should be modulate the delivery of the drug ${ }^{[1]}$. Coating fertilizer granules based on phenol or urea-formaldehyde, urea or furfuril alcohol, urethane, epoxyresins have been employed ${ }^{[1]}$. Controlled-release urea fertilizers based on acrylamide copolymers was evaluated by Abraham et al. ${ }^{[30]}$.

Acrylamide was crosslinked with comonomers with different chemical properties, such as divynilbenzene (DVB), N,N'-methylenbisacrylamide (NNMBA), tetraethyleneglycol diacrylate (TEGDA), and pentaerythrytol triacrylate (PETA) as crosslinking agents, in order to provide properties like rigidity and proper hydrophobic-hydrophilic balance. The release experiments showed that the slow-release property is maximum for the TEGDA containing system. Rudzinsky et al. instead proposed $\mathrm{pH}$-responsive hydrogels for the encapsulation of a water insoluble pesticide and a water soluble micronutrient, such as cypermethrin and cupric sulphate respectively, based on acrylic monomers ${ }^{[31]}$.

Copolymers, with different hydrophilic mechanical and thermodynamic properties, based on methyl methacrylate (MMA), methacrylic acid (MAA) and 2hydroxyethyl methacrylate (HEMA), using ethylene glycol dimethacrylate as crosslinker, were prepared by either a solution or bulk polymerization. 
Dynamic swelling and equilibrium studies, varying $\mathrm{pH}$ conditions, indicated a $\mathrm{pH}$ dependency of water uptake properties. Swelling was higher in alkaline medium than in water or acidic media (Figure 5).

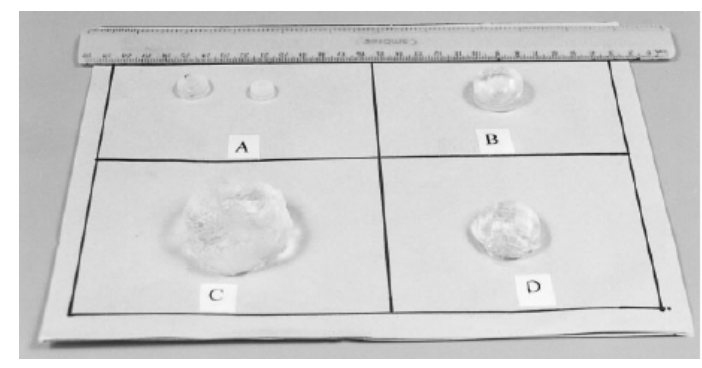

Fig. 5: Photographs showing different hydrophilicities at $30^{\circ} \mathrm{C}$ in $0.1 \mathrm{~N} \mathrm{NaOH}$ : (A) polymer samples in their dry state, (B)Polymer I, (C) Polymer III, and (D) Polymer III.

Encapsulation of cypermethrin and cupric sulfate was possible in all the hydrogels synthesized. The in vitro release experiments showed that of $\mathrm{Cu}^{2+}$ release depends on the hydrophilicity of polymer sample and nature of incapsulation solvent (Acetone, DMSO, aqueous $\mathrm{NH}_{4} \mathrm{OH}$ ), while it is not affected by the chemical interaction between the cupric ions and the polymeric structure, and the release of cypermethrin depends upon the nature of the solute as well as the rigidity of the polymeric matrix. An important class of agrochemicals delivery devices are the superabsorbent polymers, able to absorb large amounts of waters. A superabsorbent polymer with slow-release phosphate fertilizer was prepared by esterification of poly-vinyl alcohol (PVA) and phosphoric acid (SAPSRPF) by Zhan et. al. PVA and phosphoric acid react in water at $80^{\circ} \mathrm{C}$ and carried out to a mixture of polyvinyl alcohol mono- and diphosphate, (PVA-P and PVA-DP respectively) under granular shape (Figure 6) ${ }^{[32]}$.

The authors reported on the effects of the reaction time, the amount of $\mathrm{Na}_{2} \mathrm{CO}_{3}$ employed in the precipitation of polymer, the reaction temperature and the concentration of phosphoric acid on water affinity (WA) of SAPSRPF. The release profile depicted showed that the total amount of phosphate was released out in 28 day. The phosphate amount (47\%) released out in the previous 72 hours is due to fast dissolution of the fertilizer on the SAPSRPF surface layer The authors demonstrated that the PVA hydrogel decrease the water evaporation in the soil, and at the same time release the phosphate content gradually. The authors suggested this water-absorbing hydrogels suitable to reduce the loss of water by evaporation, and at the same time to release the active agents in order to improve the life span and quality of plants in dry arid areas.
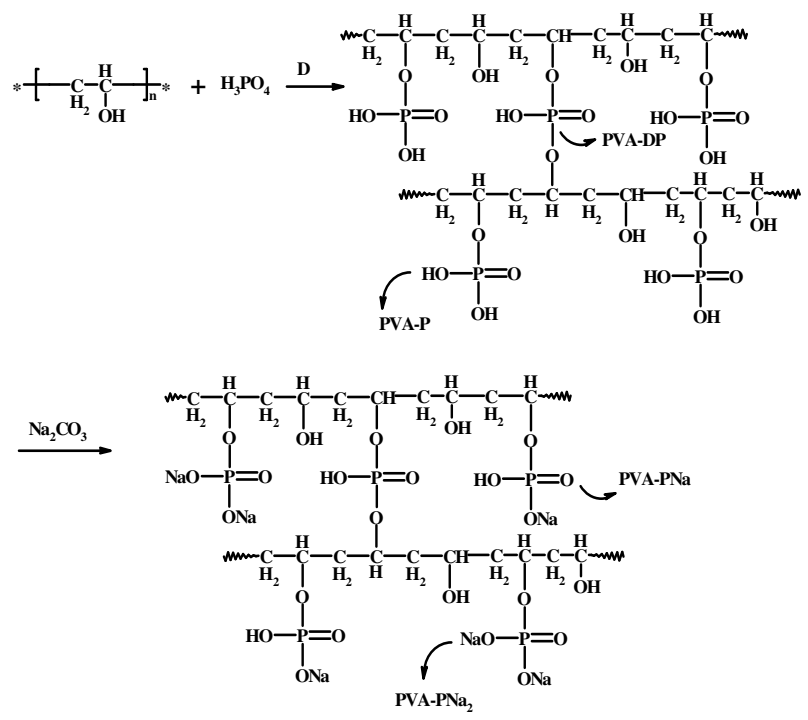

Fig. 6: Preparation of PVA-P and PVA-DP

Guo et al. described another superabsorbent and release devices on the preparation for the slow-release membrane-encapsulated urea fertilizer with superabsorbent and moisture preservation $(\text { SMUSMP })^{[33]}$. SMUSMP granules were formed by a core of urea, coated by a crosslinked starch (first layer) and a copolymer of acrylic acid (AAc) and acrylamide (AA) (second layer) (Figure 7).

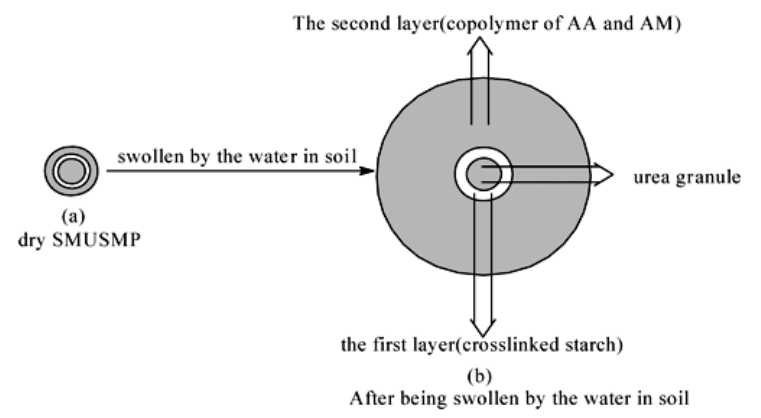

Fig. 7: Swelling Profile for SMUSMP in Soil

On the basis of the same mechanism, a coated nitrogen fertilizer with slow release and water retention (CNSW), was prepared by Liang et $\mathrm{al}^{[34]}$. The authors described the preparation of urea granules (core) coated by an inner layer based on urea-formaldehyde (UF) and by a cross-linked poly(acrylic acid)/ organ-attapulgite (O-APT) as outer layer (Figure 8). 


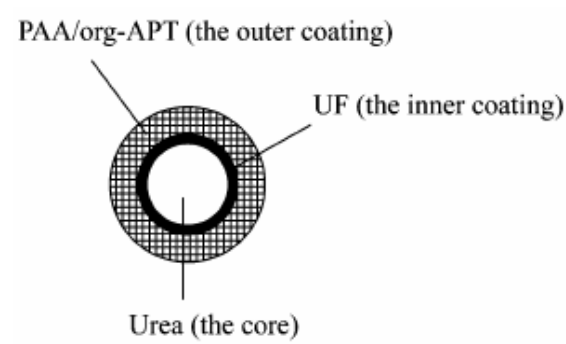

Fig. 8: Cross-sectional schematic view of a CNSW fertilizer granule

Urea-formaldehyde granules coated by a crosslinked calcium alginate (inner layer) and by a superabsorbent polymer based on ammonium acrylate (outsider layer) were developed as fertilizer with slow release, superabsorbent and moisture preservation properties (USFSMP) ${ }^{[35]}$. Du et al. studied the differential release rate and patterns of nitrate, ammonium, potassium and phosphate from two polyurethane-coated controlled release fertilizer (CRFs) and the effect of the temperature, the water content and type of release medium on it ${ }^{[36]}$. A superabsorbent with slow release nitrogen fertilizer (SSRNF) was prepared by inverse suspension polymerization of partially neutralized acrylic acid using $N, N^{\prime}$-methylene bisacrylamide as a crosslinker and ammonium persulfate as an initiator in the presence of urea ${ }^{[37]}$.

All these superabsorbent polymers showed excellent water absorbency, water retention, moisture preservation besides its slow-release properties and generally the water absorbency is strongly influenced by the amount of initiator, crosslinker, and the degree of neutralization of acrylic acid. Moreover the release ratio of the effective nutrient in it was not above $75 \%$ on the 30th day.

Biocide Polymers : As described before, one of the main problem in the use of conventional biocides is the extra amount of these compounds respect to the required one. This is due to the need to compensate the amount waste by environmental agents (rain, sun, wind etc. $)^{[38,39]}$.

In order to solve this undesirable side effects either to the plant or to public health, polymeric biocides were synthesized. Indeed, functionalized polymers containing biocidal moieties offer several advantages respect to traditional herbicides, bactericides etc..

The polymeric biocides are more persistent and do not leach out any toxic chemicals. Furthermore the size of the polymers make their incorporation in the food chain very difficult.

Biocides polymers could be incorporated into textile fibers, and used for contact disinfectant in many agri-food applications such as clothing but also as cartridge filter of potable or irrigation water. One of most important class of biocide polymers is the quaternary ammonium salt (QAS) one. Polymers containing QAS with at least a long alkyl chain (number of carbon atom $\geq 8$ ) are usually very effective against a large spectrum of microorganism such as bacteria, algae, etc. The polymers are believed to be adsorbed onto the negatively charged cell surfaces by electrostatic interaction, followed by the diffusion of the long alkyl chain through the cell wall. This results in a weakening of the cytoplasmic membrane, leading to a leakage of cytoplasmic contents and eventual death of the cell ${ }^{[40]}$. Chen et al. reported the synthesis of functionalized poly(propyleneimine) dendrimers evaluated their antibacterial properties (Figure 9) ${ }^{[41]}$.

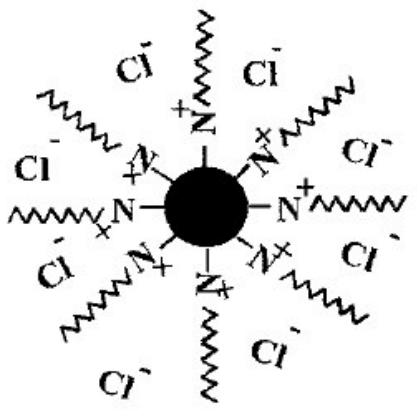

Fig. 9: Scheme of generation 2 poly(propyleneimine) dendrimer quaternary ammonium biocides with 8 QAC groups on the surface. These quaternary ammonium dendrimers are very powerful biocides. The antibacterial properties depend on the size of the dendrimer, the length of hydrophobic chains in the quaternary ammonium groups, and the counteranion. Since these dendrimers are well characterized and monodisperse, they also serve as an effective system to study the structure-activity relationship. The antimicrobial properties of these dendrimer biocides have a parabolic dependence on molecular weight, which is different from the bell-shaped molecular weight dependence of conventional polymer biocides. Lee et al. prepared a new polymeric quaternary ammonium salts via coupling reaction between the dimethylamino group of N,N-dimethyl-3-aminophenol (DMAP) and alkyl halide group of the polymer (Figure $10)^{[42]}$. 
This polymer seems to be of interest because of antibacterial activity due to its QAS in addition to the phenol group. It exhibits a stronger antibacterial activity than free DMAP. Microbeads based on quaternization of poly (4-vinyl pyridine) were reported by $\mathrm{Hu}$ et al..

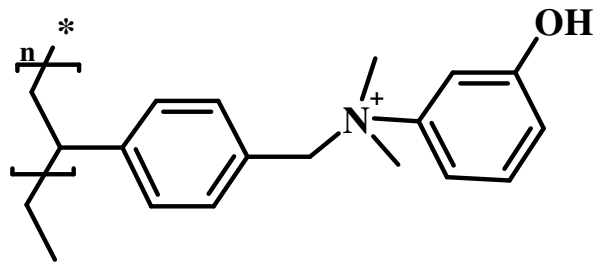

Fig. 10: Ammonium salt polymeric biocide

They showed good antibacterial and antifungal properties and could be successfully utilized for application in the areas related to biomedical, environmental protection, and water treatment ${ }^{[43]}$. Instead Kanazawa et al. reported a new classes of polymeric biocides characterized by Phosphonium and Sulfonium salts (Figure 11).
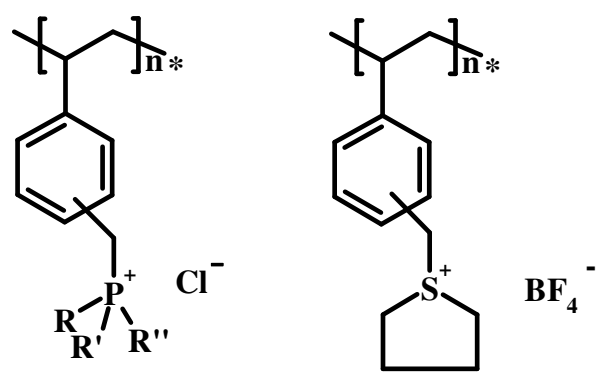

Fig. 11: Phosphonium and Sulfonium salts polymeric biocides

The antibacterial activities of these polymers were examined against E.coli and S.aureus (Gram-positive and Gram negative respectively) by the viable cell counting method in sterilized distilled water ${ }^{[44,45]}$. These studies also regarding various polymeric Phosphonium and Sulfonium Salts containing long alkyl chains and their model compounds which possess the same hydrophobic structure as that of the common disinfectants (QAS). The polymer with the decyl group exhibited a higher activity than that of the corresponding model compound, particularly against S.aurus. Although polymeric phosphonium salts exhibit a bactericide properties both Gram-positive and Grma negative, polymers with sulfonium salts exhibited only a high antibacterial activity against Gram-positive bacteria and it was found that their activity increased with an increase in MW. Recently, novel N-halamines polymers have attracted considerable interest in the polymeric biocides area ${ }^{[46]}$.

An $N$-halamine may be defined as a compound containing one or more nitrogen-halogen covalent bonds (Figure 12).

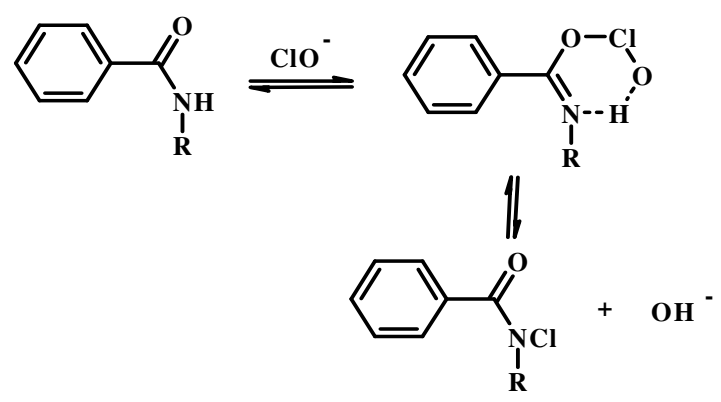

Fig. 12: synthesis of $N$-halamine polymeric biocide

The biocidal action of $N$-halamines was believed to be a manifestation of a chemical reaction involving the direct transfer of positive halogens from the $\mathrm{N}$ halamines to appropriate receptors and/or following oxidative reactions in the microorganism cells. This transfer reaction could effectively destroy or inhibit enzymatic or metabolic cell processes, consequently resulting in the expiration of the organisms ${ }^{[47]}$. Moreover, after the antimicrobial activity is lost because of extensive usage, the antimicrobial functions could be regenerated by simply treating the corresponding materials with halogen-releasing agents again. Therefore, $N$-halamines are powerful and refreshable biocides against a wide spectrum of microorganisms ${ }^{[48]}$. Although several stable watersoluble monomers were developed that possessed excellent biocidal properties, the commercialization of these compounds has been impossible because of the expensive, exhaustive toxicity testing that must be carried out to gain regulatory approval for new compounds. However an insoluble polymeric biocide might obtain approval for a biocidal water-filter application with less expensive testing if it could be shown that not significant amounts of chemicals are leached into water flowing through it. Generally in order to prepare them, two approaches have been employed - polymerization of unsaturated side chains on biocidal monomers and modification of commercial polymers by introduction of biocidal moieties. Sun et al. proposed modifications of commercial polyamides (Kevlar, Nomex, etc.) via direct N-chlorination ${ }^{[49]}$. These N-halamine provided powerful, durable and regenerable antibacterial against both Gram-negative and Gram-positive bacteria. 
The same authors studied a synthesis of polymers based on imidazolidin-4-one and hydantoin derivatives monomers. After chlorine bleach treatment, hydantoin and Imidazolidin-4-one units in the grafted copolymers were transformed into $\mathrm{N}$-halamine structures. The antimicrobial efficacies and stabilities of the polymeric $N$-halamines could be rationalized by the hydrophilic/hydrophobic property of the substrates and mobility/swellability of the polymeric molecules, as well as the surface area of the materials and migration of potentially active chlorine. The hydrophobic characteristic of synthetic fabrics had a great influence on their antibacterial properties ${ }^{[50]}$. Because the aqueous bacterial suspension could not make sufficient surface contact with the fabrics, a longer contact time was necessary for satisfactory antibacterial results. Other types of polymenc biocides that have recently been prepared and studied; Oh et al. and coworkers have prepared several series of copolymers and blends, which could be classified as benzimidazoles, polyethers and polyesters ${ }^{[51]}$. The materials appear to have potential as fungicidal and bactericidal films, but the monomers from which the polymers are produced seem to have greater activity than the polymers. other recent biocidal polymers that have been prepared and evaluated for antimicrobial efficacy include poly(hexamethylene guanidine) reacted with poly(acrylic acid) and grafted onto cellulose, polyacrylate with biguanides pendant groups ${ }^{[52]}$. Rojo and coworkers studied modifications of the chemical structure of eugenol by the incorporation of a polymerizable group, e.g., the methacrylic group ${ }^{[53]}$. This approach allows the eugenol derivatives to participate in polymerization reactions rather than to inhibit them. The new derivatives could be incorporated to permanent restorative materials, giving to the macromolecular chains the bactericide effects of eugenol.

\section{POLYMERS FOR HEAVY METALS REMOVAL}

Soil pollution has become an important environmental issue due to changes in the land use pattern (urban-industrial expansion, infrastructure development, and tourism increase). The soil is a longterm sink for the group of potentially toxic elements often referred to as heavy metals, including zinc ( $\mathrm{Zn})$, copper $(\mathrm{Cu})$, nickel $(\mathrm{Ni})$, lead $(\mathrm{Pb})$, chromium $(\mathrm{Cr})$ cadmium (Cd), mercury (Hg) and arsenic (As). The concentrations of these metals in soils are associated with biological and geochemical cycles and are influenced by human activities, such as agricultural practices, transport (automotive, aerospace), industrial activities (miming, metallurgy, chemical), and water and wastewater streams. As a consequence of these activities, a concentration of these toxic metals in the biosphere is created; thus there is ample opportunity for exposure to toxic metals ${ }^{[54]}$. Considering the harmful effects of heavy metals, it is necessary to almost totally remove them from waste effluents ${ }^{[55]}$, for this reason all over the world industry is forced to diminish down the acceptable level contents of heavy metal in water and industrial wastewaters ${ }^{[56]}$. When soils are contaminated with heavy metals, the clean-up is one of the most difficult tasks for environmental engineering. For remediating sites contaminated with inorganic pollutants, several techniques have been developed. All these technique are based on two principles referred to as immobilization or mobilization principle ${ }^{[57]}$. In the first case, the retention of heavy metals on soil is increased, but in this way a not permanent solution is raised, because metals still remain in the soil with possible long-term side effect on bio-systems. The mobilization methods, which is recently become the most common technique for soil decontamination, involve the removal of metals from the soil matrix using various washing solution agents. Generally, the conventional processes to treat contaminated water are chemical precipitation, electrodeposition, crystallization, evaporation, liquid-liquid extraction and membrane processes such as electrodialysis (ED), nanofiltration (NF) and reverse osmosis (RO). However, when contaminants are in low concentrations, these methods can become inefficient ${ }^{[58]}$. Furthermore, waste solutions generally contain large quantities of various ions which are hazardous for the environment, and selective removal of only one kind of ions is needed. One way to obtain the selective decontamination of effluent streams is to employ of polymeric ion exchange resins, with the replacement of the undesirable ion by another one which is neutral within environment ${ }^{[56]}$. Generally, the synthetic ionexchange resins are very versatile materials based on crossilnked polymers with various geometric pore structures (microporous, mesoporous, and macroporous $)^{[59]}$. The most common crosslinking agents are divinylbenzene ${ }^{[60]}$ and ethylene glycol dimethacrylate (EGDMA) ${ }^{[61]}$, while many different surface functionalities are raised by using comonomers with the desired functional groups at the stage of copolymerization or by chemical transformation of the formed polymer using appropriate modifying agents. For the preparation of this kind of materials, the most common functional groups responsible of the 
interaction with the metal ions are styrene ${ }^{[62]}$ and weakly acidic or basic functionalities: in particular a resin based on acrylic acid was employed by Kocaoba et al. ${ }^{[63]}$ for $\mathrm{Cr}$ (III) removal, acrylamide (AAm)-based hydrogel with different water absorption properties (Figure 13) are very useful in $\mathrm{Pb}$ (II), $\mathrm{Hg}$ (II), Cd (II) and $\mathrm{Cr}$ (VI) removal ${ }^{[64,65]}$ and amine resins for $\mathrm{Cr}$ $(\mathrm{VI})^{[66]}, \mathrm{Ni}(\mathrm{II}), \mathrm{Zn}(\mathrm{II}), \mathrm{Cu}(\mathrm{II}), \mathrm{Cd}(\mathrm{II})$ and $\mathrm{Pb}(\mathrm{II})^{[67]}$.

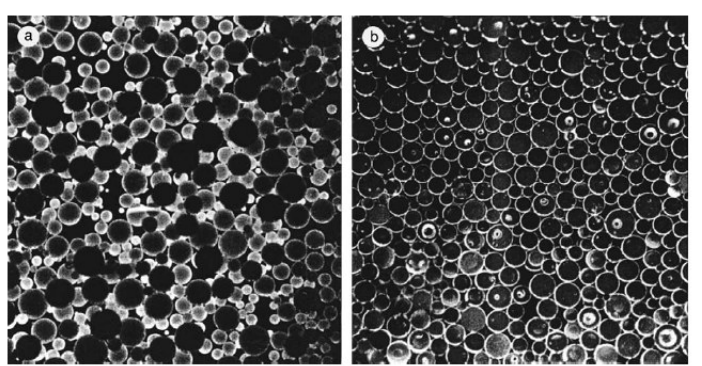

Fig. 13: Optical micrograph of poly(EGDMA-coAAm) beads: a) dry, and b) wet state.

Several articles showed that others versatile functionalities are sulfonate groups and phosphoric groups. ${ }^{[68,69]}$. They were able to create thermosensitive resins with LCST able to bind $\mathrm{Cu}$ (II) ions; finally thiacrown polymers immobilized on polystyrenedivinylbenzene were employed for $\mathrm{Hg}$ (II) removal ${ }^{[70]}$. The remotion of metals pollulant was also performed by solid phase extraction (SPE) technique. SPE technique is based on the distribution of analyte between aqueous solution and sorbent by mechanisms, such as adsorption, co-precipitation, complex formation and other chemical reactions on or in the sorbents. A new approach to this methodology has been developed using Ion Imprinted Polymers (IIPs) as sorbents to join high molecular recognition properties to the separation technique. Like Molecularly Imprinted Polymers (MIPs), IIPs are materials with high recognition properties toward the template molecules around which they have been synthesized. In IIPs case, the template is a metal ion, and with this technique polymers for the selective removal heavy metals ions have been successfully synthesized. Important results were reported for $\mathrm{Pb}$ (II) (Figure 14), (Cd (II) and $\mathrm{Co}$ (II) removal.

In recent years, attention has focused on the application of cost effective alternative technologies or sorbents for treatment of metals contaminated waste streams. Natural materials that are available in large quantities, or certain waste products from industrial or agricultural operations, may have potential as inexpensive sorbents, thus several product including carbonaceous materials, agricultural by-products have been widely studied for metal removal from water.

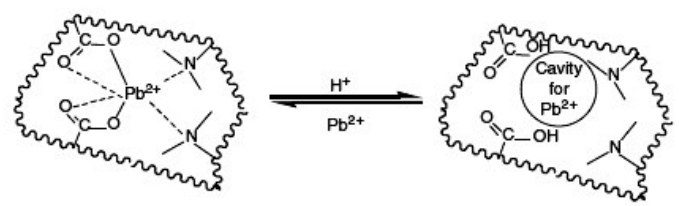

Fig. 14: Adsorption-desorption mechanism of $\mathrm{Pb}$ (II) imprinted polymers.

These include peat, wood, pine bark, banana pith, rice bran, soybean and cottonseed hulls, etc.. ${ }^{[71]}$. Most of these works have shown that plant material could replace water insoluble support rather than the typical water insoluble, synthetic polymers made from polystyrene and divinylbenzene polymer resins, activated carbon, activated alumina, which are often expensive and non-regenerable. Unfortunately, lignocellulosic material in the form of various agricultural by-products possesses little ability to bind cations and anions found in solution. In order to enhance this ability, cationic or anionic groups must be added to the particular by-product, e.g. cellulosecontaining agricultural by-products modified with the cross-linking reagent dimethyloldihydroxyethylene urea and the quaternary amine ${ }^{[72]}$. In this way, resins with a dual functionality for both cations and anions were prepared by two separate reaction of by-products. In the first one, the cross-linking reagent dimethyloldihydroxyethylene urea and a quaternary amine (choline chloride) were employed to add positive charge to the lignocellulosic material. In the second two-step process, the order of reaction was reversed, with positive charge added first, followed by the addition of negative charge. These combined reactions added both cationic and anionic character to the byproducts as evidenced by the increased removal capacity towards copper cations and chromate anions.

One of the most abundant and interesting biopolymers which can be applied for the recovery of heavy metal ions from industrial effluents is chitin, and in particular chitosan, the polymer obtained by its alkaline deacylation. The main commercial sources of chitin are crab and shrimp shells, though other sources such as fungal biomass, insect cuticle or squid pen may be used $^{[73]}$. In chitosan structure, many amine groups can be observed, due to the presence of acetylglucosamine and glucosamine units. This functionalities are responsible for the uptake of metal cations by a chelation mechanism. Indeed, nitrogen atoms hold free electron doublets that can react with metal cations. 
Furthermore, the amine groups are easily protonated in acidic solutions, carrying out the formation of electrostatic attraction of anionic compounds, including metal anions or anionic dyes ${ }^{[74]}$. With physical modification, it is possible to prepare differently conditioned polymer forms such as powder, nano-particles, gel beads, membranes, sponge, honeycomb, fibers or hollow fibers for varied fields of application (waste water treatment, biomedical, textiles, etc.). Chemical modifications are represented by the crossinking of chitosan with opportune agents ${ }^{[75]}$ such as glutaraldehyde, 1,1,3,3-tetramethoxypropane, oxidized-cyclodextrin, ethyleneglycol diglycidyl ether, or hexamethylenediisocyanate. A particular chemical modification of chitosan involves the imprinting method, which allows to increased sorption capacity for metals such as rhodium, copper and gallium ${ }^{[74]}$. The selected metal ions is adsorbed prior to the chemical modification, which may consist of a chemical grafting or cross-linking step. After this step, a cavity tailored to the volumetric space of the target metal for further sorption operation. Finally, derivatized chitosan is a base material to prepare Ion Imprinted Polymers, as demonstred in Sun et al. work ${ }^{[76]}$. Starch-based polymers are another group of useful low-cost sorbent for heavy metals, in particular in Delval et al. work ${ }^{[77]} \mathrm{a}$ very effective starch-based polymer was prepared with a crosslinking reaction between starch-enriched flour and epichlorohydrin as crosslinking agent in the presence of $\mathrm{NH}_{4} \mathrm{OH}$. Cork is also effective in heavy metals removal by virtue of its interaction with $\mathrm{Ni}(\mathrm{II})$ and $\mathrm{Cu}$ (II) ions, as reported by Villaescusa et al. ${ }^{[78]}$.

Another technique for removal of metal ions from wastewaters is the use of functionalized water-soluble polymers combined with the membrane-based separation method of ultrafiltration ${ }^{[79]}$. It consists in making heavy metals react with a water-soluble macromolecular ligand to form a macromolecular complex. Solution containing macromolecular complex is pumped through an ultrafiltration membrane. Unbound chelates pass through the membrane, while metal-loaded polymers are of sufficient molecular size to be retained. The advantages of Polymer Filtration over others metal ion separation techniques are the following: 1) high selectivity for the metal ion to be separated, 2) high reaction rate for the sorption of the metal ions on the bonding agents, 3) the possibility to employ commercially available polymers modified to selectively bind the target metal ions 4) The large quantities of wastewater can be treated in a short period of time, with a comparatively low investment in equipment. The most common water soluble polymer employed in this technique is polyethylenimine, a highly branched aliphatic polyamine. This macromolecules is useful in mercury removal: Sauer et al. ${ }^{[80]}$ reported that when it is functionalized with bromo- or chloroacetic acid to give an aminocarboxylate chelating group, it is able to effectively binds lead with binding constants similar to the molecular chelator ethylenediaminetetraacetic acid. By functionalization with sulphur donors, Gohdes et al. ${ }^{[81]}$ were able to prepare a polymers with loading capacities substantially higher than those for the base one.

\section{SUPERABSORBENT POLYMERS}

The water lack and the desertification are very serious problems for many regions of the world because, first of all, they compromise agriculture development.

Desertification is the degradation of land in arid, semi arid and dry areas resulting from various factors including climatic variations, but primarly human activities. A valid aim to these problems could come from the use of synthetic materials with good water absorption and retention capacities even under high pressure or temperature. Systems of this type are the Superabsorbent polymers (SAPs). Because of their excellent properties, these SAPs were already well established in various applications such as disposable diapers, hygienic napkins, cement, drug delivery systems, sensors, and agriculture. In such applications, water absorbency and water retention are essentials. Their use for agricultural applications has shown encouraging results; they have been observed to help reduce irrigation water consumption and the death rate of plants, improve fertilizer retention in the soil, and increase plant growth rate. Recent articles reported the modification of these superabsorbent copolymers with a view to enhance their absorbency, gel strength, and absorption rate. Raju et al. ${ }^{[82]}$ prepared a series of superabsorbent copolymers by using acrylamide (Am), potassium methacrylate (KMA) and 2hydroxyethilmethacrylate (HEMA) as monomers, and $\mathrm{N}, \mathrm{N}$-methylenebisacrylamide (MNBA) as a crosslinking agent. The influence of various synthetic parameters such as the monomer concentration, crosslinker concentration, and initiator concentration were studied. The experimental results showed that these superabsorbents polymers have a good absorbency both in water and $\mathrm{NaCl}$ solutions and a fast swelling capacity. Furthermore, it was observed that SAPs determine a considerable increase of the water 
retention of the soil; in their presence, besides, the germination energy of groundnut seeds and growth of young plants enhanced enormously. In order to obtain more easily biodegradable SAPs and reduce the product cost, Chen and al. ${ }^{[83]}$ prepared novel polymers by grafting copolymerization of acrylic acid and acrylamide onto starch by using $\gamma$-ray radiation technique and poly(ethilene glycole) (PEG) as a crosslinker. Also in this case, the effect of various synthetic parameters (irradiation dose, irradiation dose rate, monomer concentration, monomer/starch ratio, and PEG content) and the effects of different drying methods on water absorbency of the SAPs were studied. From this study turned out that, with increasing of the monomer concentration, the water absorbency decreases rapidly. This happen because high monomer concentration increase the polymer density and, consequently, also its capacity of retaining the heat produced by polymerization. As a consequence, an increase of the system temperature accelerate the chain termination and transfer speeds. On the contrary, the water absorbency increases rapidly when the PEG concentration in the polymer increases, but reaches saturation at about $0.65 \mathrm{wt} \%$ PEG. Furthermore, this SAPs show good water retention at high temperature and this property makes them suitables for a potential application in agriculture, especially in arid and desert regions. By adding a small percentage of SAPs (0.1 wt $\%)$ to sand and soil samples, in fact, the water retention of sand and soil was considerably enhanced. Finally, the effects of the SAPs on the germination of corn seeds and growth of young plants were investigated, showing that not only the germination energy of the seeds with SAPs is higher than that of the seeds without SAPs, but the superabsorbent polymers also promote the seeds growth and have a favorable effects on the weights of leafages and roots of the plants. Polyacrylate superabsorbents generally exhibit a very high absorbency in deionized water; they, however, have the problem of poor resistance to salts as evinced by their notable low absorbency exhibited to electrolytic solutions such as an aqueous common salt solution. In order to enhance the water absorbency properties of SAPs in such electrolytic solutions, Ma et al. ${ }^{[84]}$ prepared salt-resistant superabsorbent as follows. At first, they prepared crosslinked sodium polyacrylate; then, they used ethylene glycol diglycidyl ether (EGDE) to crosslink the molecular chains existing at least in the vicinity of the surfaces of the crosslinked sodium polyacrylate; last, the surface-crosslinked superabsorbent was mixed with inorganic salt powders.
The experimental data showed that the water absorbency first increased with increasing reaction temperature, neutralization degree of acrylic acid, amount of initiator, crosslinking agent, and surfacecrosslinking agent. In recent years, superabsorbent polymers prepared from natural polymers such as starch $^{[85]}$, chitosan ${ }^{[86]}$, a high molecular weight polysaccharide from chitin, and poly(amino acid) ${ }^{[87]}$ have received increasing interest because they are environmentally friendly, biodegradable, and independent of soil resources. Zhang et al. ${ }^{[88]}$ prepared a novel superabsorbent composite through graft polymerization with chitosan, acrylic acid and attapulgite in aqueous solution, using N,Nmethylenebisacrylamide (MNBA) as a crosslinker and ammonium persulfate as an initiator. Cellulose and its derivatives are also attracting a great deal of interest again for preparing superabsorbent polymers because of their biodegradable characteristics ${ }^{[89,90]}$, their natural abundance, and potentially high absorption properties $^{[91]}$. Many efforts have been made to synthesize cellulose-based superabsorbents and to improve the swelling capacity. For example, Kuwabara and Kubota ${ }^{[92]}$ synthesized highly water-absorbing acrylamide (AA) grafted carboxymethylcellulose (CMC) by photografting in the presence of $\mathrm{N}, \mathrm{N}^{1}$ methylenebisacrylamide (MNBA) as a crosslinker. Yoshinobu et al. ${ }^{[93]}$ reported partially hydrolyzed graft copolymers of crosslinked polyacrylamide on cellulose and its derivatives synthesized by a ceric salt initiation method. Kubota and Kuwabara ${ }^{[94]}$ prepared AA-grafted and methacrylic acid grafted CMC superabsorbents by photoinitiation, ceric salt initiation, and radiation initiation, and the highest water absorbency obtained was less than $250 \mathrm{~g} / \mathrm{g}$. Lionetto et al. prepared a superabsorbent cellulose-based hydrogel by crosslinking a sodium salt of CMC and hydroxyethyl cellulose with divinyl sulfone as a crosslinker.

Suo et al. ${ }^{[95]}$, instead, attempted to synthesize a new cellulose-based superabsorbent by simultaneously grafting two kinds of hydrophilic monomers, acrylic acid and acrylamide (Am), onto CMC in the presence of the crosslinker NMBA and potassium persulfate and sodium metabisulfite (PPS/SMB) as a redox couple of the initiator. The initiator and crosslinker contents, and bath temperature mainly affect the molecular weight and crosslinking density of the copolymer and the polymerization rate. The greater the weight percentage of the AA monomer in the starting stock is, the more numerous the ionic carboxylate groups are, and this greatly improves the water absorbency. The preparation of polymer/clay superabsorbent composites has also 
received great attention because of their relative low production costs and high water absorbency. Li and Wang ${ }^{[96]}$ prepared superabsorbent composites by graft copolymerization reaction of acylic acid (AA) and acrylamide (Am) on attapulgite micropowder using $\mathrm{N}, \mathrm{N}$-methylene bisacrylamide (MBA) as a crosslinker and ammonium persulphate (APS) as an initiator in an aqueous solution. Acrylamide is a kind of nonionic monomer and has great advantage on its good saltresistant performance as a raw material for superabsorbent. Attapulgite, as a good substrate for superabsorbent composite materials, is a layered aluminium silicate with reactive groups $-\mathrm{OH}$ on the surface. Also in this study, the effects of saline solutions, amount of initiator, crosslinker and attapulgite on the water absorbency of superabsorbent composites were investigated. The water absorbencies in distilled water and in $0.9 \% \mathrm{NaCl}$ solution were both improved and the production cost was significant reduced by compared with crosslinked poly (acrylic acid-co-acrylamide) superabsorbent polymer. This excellent water absorbency and water retention may prove especially practical in agricultural and horticultural applications.

\section{REFERENCES}

1. Akelah, A. 1990. Applications of functionalized polymers in agriculture. - J. Islam. Acad. Sci. 3 (1): 49-61.

2. Akelah A., E.R. Kenawy and D.C. Sherrington, 1995. Hydrolytic release of herbicides from modified polyamides of tartrate derivatives. - Eur. Polym. J. 31 (9): 903-909.

3. Bastioli, C., 1998. Biodegradable materials Present situation and future perspectives. Macromol. Symp. 135: 193-204.

4. Petruzzelli, D., A. Volpe, A.C. Di Pinto and R. Passino, 2000. Conservative technologies for environmental protection based on the use of reactive polymers. - React. Funct. Polym., 45: 95-107.

5. Nayak, P.L., 1999. Biodegradable polymers: Opportunities and challenges. - Rev. Macromol. Chem. Phys. 39: 481-505.

6. Wang, X.L., K.K. Yang and Y.Z.Wang, 2003. Properties of starch blends with biodegradable polymers. - J Macromol Sci - Pol. Rev. 43: 385409.

7. Gross R.A. and B. Kalra, 2002. Biodegradable Polymers for the Environment. - Green Chem. 297: 803-807.
8. Olabisi, O., L.M. Robinson and M.T. Show, 1979. Polymer-Polymer Miscibility. - Academic press: New York.

9. Xiao, C., Y. Lu, H. Liu and L. Zhang, 2000. Preparation and Characterization of Blend Films of Poly(Vinyl Alcohol) and Sodium Alginate. - J. Macromol. Sci. A 37: 1663-1675.

10. Chadra, R. and R. Rustgi, 1998. Biodegradable polymers. - Prog. Polym. Sci. 23: 1273-1335.

11. Halley, P., R. Rutgers, S. Coombs, J. Gralton, M. Jenkins, H. Beh, K. Griffin, R. Jayasekara, and G. Lonergan, 2001. Developing biodegradable mulch films from starch-based polymers. - Starch 53: 362-367.

12. Kaplan, D.L., J.M. Mayer, M. Greenberger, R.A. Gross and S. McCarthy, 1994. Degradation methods and degradation kinetics of polymerfilms. - Polym. Degrad .Stab. 45: 165-172.

13. Briassoulis, D., 2004. An overview on the mechanical behaviour of biodegradable agricultural films. - J. Polym. Environ. 12: 65-81.

14. Bastioli, C. 1998. Properties and applications of Mater-Bi starch-based materials. - Polym. Degrad. Stab. 59: 263-272.

15. Marini L. and S.A Novamont, 2001. Global Status of the Production of Biobased Materials. Actin Conference, Birmingham, UK, 29 to 30 March 2001.

16. Gross, R.A., J.-D. Gu, D. Eberiel and S. P. McCarthy, 1995. Laboratory-scale composting test methods to determine polymer biodegradability model studies on cellulose-acetate. - J. Macromol. Sci. A 32: 613-617.

17. Katan, J., A. Greenberger, H. Alon and A. Grinstein, 1976. Solar heating by polyethylene mulching for control ofdiseases caused by soil-bome pathogens. - Phytopathology 66: 683-688.

18. Katan, J., A. Grinstein, A. Greenberger, O. Yarden and J.E. DeVay, 1987. The first decade (19761986) of soil solarisation (solar heating): A chronological bibliography. - Phytoparasitica 15: 229-255.

19. Russo R., M. Malinconico, L. Petti and G. Romano, 2005. Physical behavior of biodegradable alginate-poly(vinyl alcohol) blend films. - J. Polym. Sci. Pol. Phys. 43: 1205-1213.

20. Russo R., A. Giuliani, B. Immirzi, M. Malinconico and G. Romano, 2004. Alginate/polyvinylalcohol blends for agricultural applications: Structureproperties correlation, mechanical properties and greenhouse effect evaluation. - Macromol. Symp. 218: $241-250$. 
21. Draget, K.I., G. Skjak Braek, and O. Smidsrod, 1997. Alginate based new materials. - Int. J. Biol. Macromol. 21: 47-55.

22. Grasdalen, H., B. Larsen and O. Smidsrod, 1981. ${ }^{13} \mathrm{C}$-NMR studies of alginate. - Carbohydr. Res. 89: 179-185.

23. Akelah, A., 1996. Novel utilizations of conventional agrochemicals by controlled release formulations. - Mat. Sci. Eng. C - Bio. S. 4: 8398.

24. Kenawy E.R., 1998. Biologically active polymers: controlled release formulations based on crosslinked acrylamide gel derivatives. - React. Funct. Polym. 36: 31-39.

25. Zhu Z., and R. Zhuo, 2001. Slow release behavior of starch-g-poly(vinyl alcohol) matrix for 2,4,5trichlorophenoxyacetic acid herbicide. - Eur. Polym. J. 37 (9): 1913-1919.

26. Zhu Z., R. Zhuo. 2001. Controlled Release of Carboxylic-Containing Herbicides by Starch- gpoly(butyl acrylate). - J. Appl. Polym. Sci. 81: 1535-1543.

27. Taki S., E. Badens, and G. Charbit. 2001. Controlled release system formed by supercritical anti solvent coprecipitation of a herbicide and a biodegradable polymer. - J. Supercrit. Fluid. 21 (1): 61-70.

28. Boutin O., E. Badens, E. Carretier, G. Charbit. 2004. Co-precipitation of a herbicide and biodegradable materials by the supercritical antisolvent technique. - J. Supercrit. Fluid.31: 89-99.

29. Elbahri Z. and J.L. Taverdet. 2005. Optimization of an herbicide release from ethylcellulose microspheres. - Polym. Bull. 54 (4-5): 353-363.

30. Abraham J. and V.N. Rajasekharan Pillai, 1996. Membrane-encapsulated controlled-release urea fertilizers based on acrylamide copolymers. - J. Appl. Polym. Sci. 60: 2347-2351.

31. Rudzinski W.E., T. Chipuk, A.M. Dave, S.G. Kumbar and T.M. Aminabhavi. 2003. pH-Sensitive Acrylic-Based Copolymeric Hydrogels for the Controlled Release of a Pesticide and a Micronutrient. - J. Appl. Polym. Sci. 87: 394-403.

32. Zhan F., M. Liu, M. Guo and L. Wu. 2004. Preparation of Superabsorbent Polymer with SlowRelease Phosphate Fertilizer. - J. Appl. Polym. Sci. 92: 3417-3421.

33. Guo M., M. Liu, F. Zhan and L. Wu. 2005. Preparation and properties of a slow-release membrane-encapsulated urea fertilizer with superabsorbent and moisture preservation. - Ind. Eng. Chem. Res. 44: 4206-4211.
34. Liang R. and M. Liu, 2006. Preparation and properties of coated nitrogen fertilizer with slow release and water retention. - Indian Eng. Chem. Res., 45: 8610-8616.

35. Guo M., M. Liu, R. Liang and A. Niu, 2006. Granular urea-formaldehyde slow-release fertilizer with superabsorbent and moisture preservation. - J. Appl. Polym. Sci. 99: 3230-3235.

36. Du C., J. Zhou and A. Shaviv, 2006. Release characteristics of nutrients from polymer-coated compound controlled release fertilizers. - J. Polym. Environ. 14: 223-230.

37. Liu M., R. Liang, F. Zhan, Z. Liu and A. Niu, 2007. Preparation of superabsorbent slow release nitrogen fertilizer by inverse suspension polymerization. Polym. Int. 56: 729-737.

38. Tashiro, T., 2001. Antibacterial and Bacterium Adsorbing Macromolecules. - Macromol. Mater. Eng., 286: 63-87.

39. Worley, S.D. and G. Sun, 1996. Biocidal Polymers. - Trend Polym. Sci. 4: 364-370.

40. Nurdin N., G. Helary and G. Sauvet, 1993. Biocidal polymers active by contact. II. Biological evaluation of polyurethane coatings with pendant quaternary ammonium salts. - J. Appl. Polym. Sci. 50: 663-670.

41. Chen C.Z., N.C. Beck-Tan, P. Dhurjati, T.K. van Dyk, R.A. La Rossa and S.L. Cooper, 2000. Quaternary Ammonium Functionalized Poly(propylene imine) Dendrimers as Effective Antimicrobials: Structure-Activity Studies. Biomacromolecules. 1: 473-480.

42. Lee Y.S. and Y.S.Byoun, 2002. Poly(styrene-co-4vinylbenzyl chloride) Conjugated with 3(Dimethylamino)phenol: Synthesis and Antibacterial Activity. - Bull. Korean Chem. Soc. 23: 1833-1835.

43. Hu F.X., K.G. Neoh, L. Cen and E.T. Kang, 2005. Antibacterial and antifungal efficacy of surface functionalized polymeric beads in repeated applications. - Biotechnol. Bioeng. 89: 474-484.

44. Kanazawa A., T. Ikeda and T. Endo, 1993. Polymeric phosphonium salts as a novel class of cationic biocides. III. Immobilization of phosphonium salts by surface photografting and antibacterial activity of the surface-treated polymer films. - J. Polym. Sci. Pol. Chem. 31: 1467-1472.

45. Kanazawa A., T. Ikeda and T. Endo, 1993. Antibacterial activity of polymeric sulfonium salts. - J. Polym. Sci. Pol. Chem. 31: 2873-2876. 
46. Sun G., W.B. Wheatley and S. D.Worley, 1994. A New Cyclic N-Halamine. Biocidal Polymer. Indian Eng. Chem. Res. 33:68-170.

47. Sun Y. and G. Sun, 2002. Synthesis, characterization, and antibacterial activities of novel N-halamine polymer beads prepared by suspension copolymerization. - Macromolecules. 35: 8909-8912.

48. Sun Y. and G. Sun, 2001. Novel regenerable Nhalamine polymeric biocides. I. Synthesis, characterization, and antibacterial activity of hydantoin-containing polymers. - J. Appl. Polym. Sci. 80: $2460-2467$.

49. Sun Y. and G. Sun, 2004. Novel refreshable NHalmine polymeric biocides: N-Chlorination of aromatic polyamides. - Indian Eng. Chem. Res. 43: 5015-5020.

50. Sun Y. and G. Sun, 2003 Novel refreshable NHalmine polymeric biocides: Grafting Hyndatoincontaining monomers onto High Peformances fiber by a continuous process. - J. Appl. Polym. Sci. 88: 1032-1039.

51. Oh S.T., B. K. Min, C.S. Ha and W. J. Cho, 1993. Synthesis and fungicidal activities of polymeric biocides. I. TBZ-containing monomer and polymers. - J. Appl. Polym. Sci. 52: 583-589.

52. Virnik A.D., M.A Penenzhik, MA Crishin, , I.S. Rishkina, A.B Zezin and V.B. Rogacheva, 1994. Interpolyelectrolyte reactions between polyhexamethylene guanidine and polyacrylic acid grafted on cellulose: a new method for the preparation of antimicrobial fibrous material. Cellul. Chem. Technol 28: 11-19.

53. Rojo L., B. Vazquez., J. Parra, A.L. Bravo, S. Deb and J. S. Roman, 2006. From Natural Products to Polymeric Derivatives of "Eugenol": A New Approach for Preparation of Dental Composites and Orthopedic Bone Cements. Biomacromolecules 7: 2751-2761.

54. Mico, C., L. Recatalà, M. Peris and J. Sanchez, 2006. Assessing heavy metal sources in agricultural soils of an European Mediterranean area by multivariate analysis. - Chemosphere 65 : 863-872.

55. Nicholson, F.A., S.R. Smith, B.J. Alloway, C. Carlton-Smith and B.J. Chambers, 2003 An inventory of heavy metals inputs to agricultural soils in England and Wales. - Sci. Total Environ. 311: 205-219.

56. Dabrowski, A., Z. Hubicki, P. Podkoscielny and E. Robens, 2004. Selective removal of the heavy metal ions from waters and industrial wastewaters by ion-exchange method. - Chemosphere 56: 91106.
57. Molinari, R., T. Poerio and P. Augurio, 2005. Polymer assisted ultrafiltration for copper-citric acid chelate removal from wash solutions of contaminated soil. - J. Appl. Electrochem. 35: 375380.

58. Kurniawan, T.A., G.Y.S. Chan, W.H. Lo and S. Babel, 2006. Physico-chemical treatment techniques for wastewater laden with heavy metals. - Chem. Eng. J. 118: 83-98.

59. Saha, B. and M. Streat, 2005. Adsorption of Trace Heavy Metals: Application of Surface Complexation Theory to a Macroporous Polymer and a Weakly Acidic Ion-Exchange Resin.- Indian Eng. Chem. Res. 44: 8671-8681.

60. Bekri-Abbes, I., S. Bayoudh and M. Baklouti, 2006. Converting Waste Polystyrene into Adsorbent: Potential Use in the Removal of Lead and Cadmium Ions from Aqueous Solution. - J. Polym. Environ. 14: 249-256.

61. Malc, S., C. Kavakl, A. Tuncel and B. Salih, 2005. Selective adsorption, pre-concentration and matrix elimination for the determination of $\mathrm{Pb}(\mathrm{II}), \mathrm{Cd}(\mathrm{II})$, $\mathrm{Hg}$ (II) and $\mathrm{Cr}$ (III) using 1,5,9,13tetrathiacyclohexadecane-3,11-diol anchored poly ( $p$-chloromethylstyrene-

ethyleneglycoldimethacrylate) microbeads. - Anal. Chim. Acta 550: 24-32.

62. Galan, B., D. Castaneda and I. Ortiz, 2005. Removal and recovery of $\mathrm{Cr}(\mathrm{VI})$ from polluted ground waters: A comparative study of ionexchange technologies. - Water Res. 39: 43174324.

63. Kocaoba, S. and G. Akcin, 2004. Chromium(III) Removal from Wastewaters by a Weakly Acidic Resin Containing Carboxylic Groups. - Adsorpt. Sci. Technol. 22: 401-410.

64. Kesenci, K., R. Say and A. Denizli, 2002. Removal of heavy metal ions from water by using poly(ethyleneglycol dimethacrylate-co-acrylamide) beads. - Eur. Polym. J. 38: 1443-1448.

65. Bajpai, S.K. and S. Johnson, 2006. Poly(acrylamide-co-maleic acid) Hydrogels for Removal of Cr(VI) from Aqueous Solutions, Part 1: Synthesis and Swelling Characterization. - J. Appl. Polym. Sci. 100: 2759-2769. 
66. Gode, F. and E. Pehlivan, 2005. Removal of Cr(VI) from aqueous solution by two Lewatit-anion exchange resins. - J. Hazard. Mater. B119: 175182.

67. E1 Mostapha, J., F. Jourjon, G. Le Guillanton and D. Elothmani, 2005. Removal of metal ions in aqueous solutions by organic polymers: use of a polydiphenylamine resin. - Desalination 180: 271276.

68. Pehlivan, E. and T. Altun, 2006. The study of various parameters affecting the ion exchange of $\mathrm{Cu}^{2+}, \mathrm{Zn}^{2+}, \mathrm{Ni}^{2+}, \mathrm{Cd}^{2+}$, and $\mathrm{Pb}^{2+}$ from aqueous solution on Dowex 50W synthetic resin. - J. Hazard. Mater. B134: 149-156.

69. Tokuyama, H., K. Yanagawa and S. Sakohara, 2006. Temperature swing adsorption of heavy metals on novel phosphate-type adsorbents using thermosensitive gels and/or polymers. - Sep. Purif. Technol. 50: 8-14.

70. Baumann, T.F., J.G. Reynolds and G.A. Fox, 2002. Thiacrown Polymers for Removal of Mercury from Waste Stream. - United States Patent 6,696,576.

71. Babel, S. and T.A. Kurniawan, 2003. Low-cost adsorbents for heavy metals uptake from contaminated water: a review. - J. Hazard. Mater. B97: 219-243.

72. Marshall, W.E. and L.H. Wartelle, 2006. Chromate $\left(\mathrm{CrO}_{4}{ }^{2-}\right)$ and copper $\left(\mathrm{Cu}^{2+}\right)$ adsorption by dualfunctional ion exchange resins made from agricultural by-products. - Water Res. 40: 2541 2548.

73. Crini G., 2005. Recent developments in polysaccharide-based materials used as adsorbents in wastewater treatment. - Progr. Polym. Sci. 30: 38-70.

74. Guibal, E., 2004. Interactions of metal ions with chitosan-based sorbents: a review. - Sep. Purif. Technol. 38: 43-74.

75. Atia, A.A., 2005. Studies on the interaction of mercury(II) and uranyl(II) with modified chitosan resins. - Hydrometallurgy 80: 13-22.

76. Sun, S., L. Wang, A. Wang, 2006. Adsorption properties of crosslinked carboxymethyl-chitosan resin with $\mathrm{Pb}(\mathrm{II})$ as template ions. - J. Hazard. Mater. B136: 930-937.

77. Delval, F., G. Crini, S. Bertini, C. Filiatre and G. Torri, 2005. Preparation, characterization and sorption properties of crosslinked starch-based exchangers. - Carbohyd. Polym. 60: 67-75.
78. Villaescusa, I., N. Fiol, F. Cristiani, C. Floris, S. Lai and V.M. Nurchi, 2002. Copper(II) and nickel(II) uptake from aqueous solutions by cork wastes: a NMR and potentiometric study. Polyhedron 21: 1363- 1367.

79. Canizares, P., A. Perez, R. Camarillo and J.J. Linares, 2006. Simulation of a continuous metal separation process by polymer enhanced ultrafiltration. - J. Membrane Sci. 268: 37-47.

80. Sauer, N.N., D.S. Ehler and B.L. Duran, 2004. Lead Extraction from Contaminated Soil Using Water-Soluble Polymers. - J. Environ. Eng. 130: 585-588.

81. Gohdes, J.W., B.L. Duran, N.C. Clark, T.W. Robison, B.F. Smith and N.N. Sauer, 2001. Preparation of Water-soluble Polymers Modified with Sulfur Donors for Recovery of Heavy Metals. - Sep. Sci. Technol. 36 (12): 2647-2658.

82. Raju, K.M., M.P. Raju and Y. M. Mohan, 2001. Synthesis and water absorbency of crosslinked superabsorbent polymers. - J. Appl. Polym. Sci. 85: 1795-1801.

83. Chen, P., W. Zhang, Luo W. and Y. Fang, 2004. Synthesis of superabsorbent polymers by irradiation and their applications in agriculture. -, J. Appl. Polym. Sci. 93: 1748-1755.

84. Ma, S., M. Liu and Z. Chen, 2004. Preparation and Properties of a Salt-Resistant Superabsorbent Polymer. - J. Appl. Polym. Sci. 93: 2532-2540.

85. Wu, J.H., Y.L. Wei, J.M. Lin and S.B. Lin, 2003. Study on starch-graft-acrylamide/mineral powder superabsorbert composite. - Polymer 44: 65136520 .

86. Mahdavinia, G. R., A. Pourjavadi, H. Hosseinzadeh and M. Zohuriaan, 2004. Modified chitosan 4. Superabsorbent hydrogel from poly(acrylic acid-co-acylamide) grafted chitosan with salt- and pH-responsiveness properties. Eur. Polym. J. 40: 1399-1407.

87. Kunioka, M., 2004. Biodegradable water absorbent synthesized from bacterial poly(amino acid)s. Macromol. Biosci. 4: 324-329.

88. Zhang, J., Q. Wang. and A. Wang, 2007. Synthesis and characterization of chitosan-g-poly(acrylic acid)/attapulgite superabsorbent composites. Carbohyd. Polym. 68: 367-374.

89. Lim, K.Y., K.J. Yoon and B.C. Kim, 2003. Highly absorbable lyocell fiber spun from cellulose/hydrolyzed starch-g-PAN solution in NMMO monohydrate. - Eur. Polym. J. 39: 21152120 . 
90. Sannino, A., A. Maffezzoli and L. Nicolais, 2003. Introduction of molecular spacers between the crosslinks of a cellulose-based superabsorbent hydrogel: Effects on the equilibrium sorption properties. - J. Appl. Polym. Sci. 90: 168-174.

91. Lionetto, F., A. Sannino and A. Maffezzoli, 2005. Ultrasonic monitoring of the network formation in superabsorbent cellulose based hydrogels. Polymer 46: 1796-1803.

92. Kuwabara, S. and H. Kubota, 1996. Water absorbing characteristics of acrylic acid-grafted carboxymethyl cellulose synthesized by photografting. - J. Appl. Polym. Sci. 60: 19651970.

93. Yoshinobu, M., M. Morita and I. Sakata, 1992. Porous structure and rheological properties of hydrogels of highly water- absorptive cellulose graft copolymers. - J. Appl. Polym. Sci. 45: 805812.
94. Kubota, H. and S. Kuwabara, 1997. Cellulosic absorbents for water synthesized by grafting of hydrophilic vinyl monomers on carboxymethyl cellulose. - J. Appl. Polym. Sci. 64: 2259-2263.

95. Suo, A., J. Qian, Y. Yao and W. Zhang, 2007. Synthesis and Properties of Carboxymethyl Cellulosegraft-Poly(acrylic acid-co-acrylamide)as a Novel Cellulose-Based Superabsorbent. - J. Appl. Polym. Sci. 103: 1382-1388.

96. Li, A. and W. Wang, 2005. Synthesis and properties of clay-based superabsorbent composite. - Eur. Polym. J. 41: 1630-1637. 